\title{
Diseases of the Digestive Tract: Is Prevention Possible and Feasible?
}

\author{
Juan-R. Malagelada \\ Hospital Universitari Vall d'Hebron, Universitat Autònoma de Barcelona, Barcelona, Spain
}

\section{Key Words}

Barrett's esophagus - Colon cancer prevention •

Gastroenterology, pathological conditions •

Gastroenterology, preventive strategies $\cdot$ Personalized

medicine $\cdot$ Screening, quality assurance

\begin{abstract}
Prevention is and will remain a major goal of medicine and gastroenterology. Economics will have a major influence. Preventive approaches will need to prove a favorable costbenefit ratio before they are authorized for broad implementation. Personalized medicine is just starting, but undoubtedly it will accelerate, gain relevance and become an integral part of our clinical practice. Physicians in general and gastroenterologists specifically will need to seriously contemplate retraining/refocusing to gain competence in genetic/proteomic evaluation of individuals, or risk a significant degree of obsolescence.

Copyright $\odot 2011$ S. Karger AG, Basel
\end{abstract}

\section{Introduction}

The importance of prevention in medical practice is unquestionable. This concept is encapsulated in the timesanctioned aphorism: prevention is better than cure. But it is also underscored by a more practical and timely version: today, preventable illnesses comprise a significant proportion of total health costs. Which is to say that if we did not actively seek to prevent what we can, the final bill in human and economic terms would be considerably higher. It is clear from this line of reasoning that gastroenterologists (and for this matter physicians at large) should not retreat from a key professional mission: prevention remains a priority task.

Having re-established that principle, we must courageously express the obvious qualms. In 2011, can we in fact prevent digestive tract disease: effectively, efficiently, in every setting, for everyone? Probably not. Moreover, if we attempt to overstretch our ambitions and efforts, we risk futility and waste. Thus, we must appeal first to common sense and objectivity to establish realistic prevention targets, avoid controversial approaches and employ utmost care to avoid embarking in costly fantasies. The latter are often displayed in the form of superficially attractive but unproven technological advances cheered by overenthusiastic colleagues, industry and even speculators!

Prevention of gastrointestinal illness is, therefore, a formidable and challenging enterprise. How should we go about it? I propose we collectively organize the task into four major sections or aspects (fig. 1). First, consider the full spectrum of preventable conditions involving the

\section{KARGER \\ Fax +4161306 1234 \\ E-Mail karger@karger.ch}

www.karger.com
(C) 2011 S. Karger AG, Basel

0257-2753/11/0292-0255\$38.00/0

Accessible online at:

www.karger.com/ddi
J.-R. Malagelada

Hospital General Vall d'Hebron, Servicio de Patología Digestiva

Paseo Vall d'Hebron 119

ES-08035 Barcelona (Spain)

E-Mail juanrmalagelada@gmail.com 
1 Take a broad look at potentially preventable medical conditions and prioritize actions based on need and probability to succeed

2 Identify and promote lifestyle changes that may have preventive value

3 Review existing instruments (chemopreventive, diagnostic, interventional); promote mature technologies; help implement them

4 Perform model analysis to evaluate economic implications and facilitate the development of cost-benefit equations

Fig. 1. How to organize our task.

\begin{tabular}{|ll|}
\hline To consider & To balance against \\
\hline Risk reduction & Social cost \\
Screening & Economic cost \\
Surveillance & Effectivity \\
Preemptive ablation & latrogeny \\
Early diagnosis & Capacity \\
\hline
\end{tabular}

Fig. 2. Preventive strategies.

digestive system and establish action priorities based on two main criteria: importance (i.e. frequency, lethality, societal impact) and probability of success. Second, identify and promote lifestyle changes that may have a preventive value. This step is important for two main reasons: (a) it is probably the most economical, as it represents primarily an educational effort, and (b) because it engages individuals who, motivated by the perspective of better health, enroll themselves in the preventive effort and help disseminate positive information and attitude to others in the community. Thirdly, it is appropriate to review existing instruments of prevention to decide which are suitable and ready for application. Furthermore, under which conditions and degree of efficiency these instruments could be introduced. Mature technologies that may offer real value should be promoted and publicized to obtain broad implementation. These decisions are mainly the charge of organizations (public or private) that incorporate the necessary experts and infrastructure to assess the constant stream of technical offers and also possess the power to organize suitable teams to take action (programmatic screening, for instance).
The fourth item pertains to the economic dimension of prevention. This is indeed a critical aspect. Before embarking or recommending preventive initiatives, we must first obtain reliable cost-benefit assessments to establish whether the investment (regardless of whether eventual payors will be governments, other organizations or individuals themselves) is commensurate to the expected benefits. At the same time we must incorporate into any new initiatives the necessary elements to measure costbenefit outcomes and to appropriately disseminate this information to help others avoid costly mistakes.

\section{Instruments and Preventive Strategies in Gastroenterology}

There are a number of available instruments of prevention that we may utilize in gastroenterology. The most important ones are risk reduction, screening, surveillance, preemptive ablation and early diagnosis (fig. 2). Risk reduction is mainly but not exclusively achieved through modifications in lifestyle: changes in diet, weight loss, exercise, avoidance of smoking, excess alcohol, drugs, etc. For an important minority of conditions, i.e. celiac disease, risk reduction also entails avoidance of specific culprit foods present in the regular diet. This process should be facilitated by actively stimulating the food industry to make commercially available and economically accessible the appropriate substitutes. New technologies to inhibit or antagonize the triggering food component are also being developed.

Screening is a prevention approach that investigates apparently healthy individuals looking for lesions or conditions susceptible of developing into a serious pathological state. The concept of screening contemplates the possibility of either applying it to a cross-segment of the general population or to a specific group of individuals with higher probability of developing a given disease.

Surveillance is another preventive method that differs from screening in that it focuses on individuals who are already known to harbor a lesion or condition susceptible of progressing into a dangerous state and that needs to be watched periodically to identify the stages when it needs treatment, including removal. Surveillance implies that treatment carries some difficulty or risk for the individual. Otherwise, it might be treated in the first place, rather than surveilled. In current times the concept of risk may be broadened to include financial risk if immediate treatment is so costly that it may be preferable to surveil and treat only individuals whose pathological condition 
progresses towards a truly dangerous stage. The concept of preemptive ablation is linked in many respects to surveillance and it is applied to lesions that can be removed at diagnosis at reasonable cost on the expectation that if allowed to evolve would become more difficult to treat effectively or pose a higher risk. Historically, early diagnosis was the primary objective of prevention on the reasoning that if a lesion could not be detected before reaching a stage of danger, at the very least had to be identified and treated before it became irreversible. Early diagnosis remains, of course, valid and important but screening and surveillance are currently prioritized.

The preventive strategies we just reviewed are all logical and commonly applied today. However, they also incorporate disadvantages that need to be balanced against their positive contribution. Even the apparently most innocuous recommendation, such as a change in lifestyle, carries some social cost as the individual is constrained to modify a habit that is pleasurable, ingrained or gregarious. The economic cost of preventive actions must also be taken into account since none is financially neutral. Their real cost, of course, has to be balanced in economic terms with the savings that it would eventually entail reversing adverse outcomes or avoiding even more costly treatments. In this regard the effectivity of a preventive initiative becomes very important in determining its efficiency, that is maximizing benefits at minimal cost. Thus, if a screening or surveillance procedure is not highly effective it may not be efficient to use it in the first place. The reverse, however, may not be necessarily true. Effective measures may not be efficient if they are too risky and/or costly.

Risk is not only a function of disease but also of treatment. Indeed, iatrogeny is welded to modern medicine and it must be feared and proactively avoided. Of course, iatrogeny has always coexisted with medical procedures, both diagnostic and therapeutic, but the multiplicity of invasive technologies and potent pharmacological tools available today have often powered effectivity at the expense of increased side effects. When considering a specific preventive strategy, the possibility of inducing harm must be balanced against the potential benefits. A riskbenefit analysis is already needed at the protocol building level.

Finally, capacity is a concept that also has to be weighted in, particularly when massive screening or surveillance efforts are planned for large populations. Therefore, the question whether the existing medical system will be able to cope with the increased demand for services should always be asked in advance. Manpower resources

Diseases of the Digestive Tract:

Is Prevention Possible and Feasible?

\begin{tabular}{|ll|}
\hline Pathogenesis & Preventive strategies \\
\hline Neoplastic & $\begin{array}{l}\text { Metaplasia } \rightarrow \text { dysplasia } \rightarrow \text { Ca sequence; } \\
\text { genetics }\end{array}$ \\
Inflammatory & Microbiota/diet modification \\
Ischemic & Vascular health improvements \\
Metabolic & Adequate nutrition; weight reduction \\
Infectious & Environment/vaccines/probiotics/ \\
& antibiotics \\
Hereditary & Genetic profiling/counseling \\
Functional & Education/coping \\
\hline
\end{tabular}

Fig. 3. Advances in prevention of gastrointestinal disease.

should be sufficient, particularly if high levels of specialization and expertise are required but also medical facilities, transportation, escort, etc. From the above considerations, it is apparent that preventive initiatives are complex and require a careful preparatory evaluation.

\section{Preventable Pathological Conditions in Gastroenterology}

Virtually all diseases affecting the digestive system are preventable, at least theoretically [1]. Obviously some are more amenable to preventive measures than others. Furthermore, when potential benefits are thoughtfully balanced against the potential disadvantages the list becomes shorter and it is readily apparent that systematic prevention campaigns and programs are reasonable only for certain pathologies. This is particularly true when screening and surveillance initiatives are considered, because of the expense involved.

Conditions that are suitable for prevention, at least to some extent, encompass a broad spectrum of pathogenesis (fig. 3). Neoplastic conditions of the gastrointestinal tract come first, because of their lethality and favorable disposition to preventive measures. In particular, genetically predisposed neoplasia and hereditary conditions allow identification of 'at-risk' populations in whom to focus preventive action. Also, the known sequence of metaplasia to dysplasia and eventually cancer, offers opportunities to intervene at the most suitable step. For neoplasia in which a predisposing infectious agent or medical condition is known (as would be for instance the case for Helicobacter gastritis, chronic ulcerative colitis or hepatocarcinoma) preventive steps may be undertaken at an even earlier stage. 
Inflammatory diseases of the gastrointestinal tract are putatively amenable to prevention via diet modification and intervention upon the microbiota. These measures can be very cost-effective when a clear culprit has been identified (i.e. gluten for celiac disease or dysbacteriosis in post-colectomy pouchitis). However, for most inflammatory conditions, prevention remains today an exciting prospect rather than a reality. Gut ischemia is another important health problem for which preventive strategies are generally of limited value. However, in selected cases, prevention may be very effective as it would be, for instance, in cases of large vessel stenosis repermeabilized via dilation or stent placement. Complementary longterm measures, such as lowering cholesterol levels and improving systemic cardiovascular performance, are of additional preventive value although their real impact is more difficult to quantitate.

Metabolic syndrome has a major impact upon the digestive system, particularly on the liver. Alcoholic and non-alcoholic steatohepatitis are major causes of cirrhosis and hepatocarcinoma. The progression and adverse consequences of metabolic syndrome can be prevented by weight reduction. Indeed, this is one of the major potential benefits of bariatric surgery. Diabetes-related gastrointestinal complications, including visceral autonomic neuropathy, are harder to prevent. However, there is evidence that poor glycemic control accelerates the advent of diabetic sequelae, suggesting that good management of diabetes offers some protection.

\section{The Basics of Colon Cancer Prevention}

The fact is that colon cancer is now the second cause of cancer death in Western countries. Faced with the magnitude of this health liability the only sensible course of action is to establish preventive initiatives. But the question remains: Which are the appropriate preventive measures to implement? To answer such an important question, a number of aspects need to be considered. Risk reduction via enforcement of healthy habits would seem the most logical measure to apply to the general population. Unfortunately, lifestyle changes such as avoidance of smoking and excess alcohol, restriction of dietary red meat, abundance of vegetables and fruits, exercise and others may be common sense and there are some supporting data on record [2], but conclusive data are not yet available. Admittedly, many of these lifestyle measures would have additional benefits (metabolic, cardiovascular and other) on the health of the general population and are indeed worth recommending.

Chemoprevention is also an appealing strategy. Requirements, however, would differ whether chemoprevention were to be applied to concrete high-risk groups (i.e. familial polyposis syndromes or even cancer-prone families) or to the general population. In the latter case, chemopreventive agents must meet very high safety marks and a favorable cost-benefit balance, a double requirement not so easy to attain. So far, chemopreventive strategies have not encountered much success. Omega-3 fatty acids reduce growth of polyps in familial polyposis. Aspirin $75 \mathrm{mg} /$ day has shown significant effectiveness [3], but burdened by increased gastrointestinal bleeding risk. Some non-steroidal anti-inflammatory agents have also shown some effectivity inhibiting growth of preneoplastic colon polyps but no widely consensuated chemopreventive strategy has been adopted, yet.

Screening for premalignant lesions and early diagnosis is another important colon cancer-preventive approach. The natural history of colon cancer development favors screening directed towards detection and elimination of premalignant lesions. Over time, screening methods have evolved from digital examination, hinting at the presence of rectal tumors to detection of occult blood in stools. This was done initially with relatively unspecific guaiac-based methods and more recently with specific immunochemical tests. Increasingly, there is a shift towards implementing the more sensitive and specific, yet more costly methods, based on morphological recognition of colonic lesions. However, occult blood detection has shown its value for large populations and holds its ground. Sigmoidoscopy and colonoscopy, CT colography, capsule endoscopy and others are rallying for first choice. Economic cost, invasiveness (iatrogeny) and manpower considerations are among the key decision elements besides the standard sensitivity and specificity indexes, when comparative analysis among these various imaging technologies is performed.

As I have insisted throughout this review, economic issues are unavoidable, particularly these days with the perspective of finite funds for public and insurance financed healthcare. However, the issue is not simply how much it will cost to prevent cancer but, with rapidly increasing cancer treatment costs, how much it will save by preventing cancer. Therefore both prevention costs and available treatment costs must be included in the calculations.

As gastroenterologists, we have a natural inclination to favor colonoscopy as the preferred screening method, 
because conventional endoscopy is part of our professional skills and current activity. For this reason it is particularly relevant to bring up the controversy that has recently arisen concerning the real effectiveness of colonoscopy. Some studies have introduced new doubts regarding the 'gold standard' position that colonoscopy has enjoyed so far in the screening method hierarchy. Costly, but unbeatable has been our professional slogan for colonoscopy and it has been well publicized through the media. This position is now being challenged on several counts. First, studies have suggested that even though colonoscopied individuals have a lower incidence and mortality from colon cancer than unscreened populations [4], the degree of protection actually attained falls short of what had been proclaimed in the past by less rigorous studies. This is probably because colonoscopy misses some lesions, particularly those located in the proximal segment of the colon: moreover, there is an important dilutional effect introduced by mortality from causes other than colon cancer in successfully screened individuals. While this dilutional effect does not diminish the preventive value of colonoscopy to individuals, it has important economic implications when calculating the economic cost 'per life saved' by colonoscopy screening programs $[5,6]$.

Part of the colonoscopy controversy involves the assessment methodology used to establish the actual value of colonoscopy screening. Long-term (over 10 years) controlled trials would theoretically be the most appropriate assessments method and this criterion generally prevails among European academics. Contrary views have nevertheless been expressed on grounds that the cost of such trials is excessive and are also hard to sustain to completion to allow valid interpretation of the results. Moreover, the focus certainly is switching from early cancer detection to removal of precancerous lesions and, on this count, colonoscopy has the advantage over competing non-invasive methods of allowing preemptive excision in the majority of instances.

To date, two major screening models employing colonoscopy have been employed: spontaneous screening and programmatic screening [7]. Spontaneous screening is the approach that prevails in the USA and takes advantage of the medical system at large. It applies to colon cancer screening the various methods (from occult blood detection to colography and colonoscopy) available to medical organizations, medical institutions, professional groups and individual physicians. By allowing spontaneous screening initiatives, financed by both the public and the private sector, spontaneous screening tends to extend fast and in the USA a substantial segment of the general population already benefits. However, it has some potential disadvantages. First, by using multiple screening approaches it hampers, to some extent, efforts to validate and evaluate the various methodologies employed. Thus, it will be quite difficult by means of spontaneous screening initiatives to establish the relative value of CT colography vs. capsule colonoscopy vs. standard colonoscopy, etc. because all may be used depending on availability, coverage and other extemporaneous reasons.

Adherence on the part of the population to spontaneous screening is a function of sociocultural status as the better informed and better covered financially tend to take preferred advantage of screening opportunities. There are also significant limitations. Capacity is one of them, since doctors performing screening techniques tend to enroll all willing and qualified individuals. Admittedly, however, the private sector tends to flexibility expand as needed.

The alternative screening approach, programmatic screening, also has a mixture of advantages and disadvantages. It is the preferential system applied in Europe. Programmatic screening almost by definition tends to be applied to limited populations, since it is conditioned by protocol requirements that in turn constrain recruitments. Investigators managing such programs often depend on budget-constrained resources usually provided by state financing. Consequently, programmatic screening extends among the general population at a slower pace than spontaneous screening. However, the former is better suited to incorporate efficacy assessment by controlled trial. Because it incentivates adherence, it covers a smaller but perhaps a more representative crosssegment of the general population. Limits are basically set by the availability of public resources and also by idiosyncrasies of various populations. In Europe for instance, public participation varies significantly from country to country, generally being higher in northern countries [8].

Since programmatic screening is better suited than spontaneous screening to be rolled out in randomized fashion, it facilitates comparison of existing screening modalities. This is of utmost importance given the relatively large number of existing and proposed screening instruments. It is obvious that rather than applying them indiscriminately, via spontaneous initiatives, these novel modalities should first be subjected to valid testing. Furthermore, as said earlier, cost issues must be taken into account to determine real effectiveness. 


\section{Quality Assurance}

Quality assurance is a very important aspect of the entire screening issue. It is a must for all screening modalities. Poor quality diminishes effectiveness and it would be incompatible with any useful and cost-effective screening initiative. In principle, high-quality screening appears to be more easily accomplished in the context of programmatic screening, with its pre-established and enforced protocol standards, than by spontaneous screening. However, newer US reimbursement strategies such as 'pay for performance' should incentivate and improve quality. In Europe, with its emphasis in programmatic screening, direct action to enhance quality is increasingly being incorporated into screening protocols. In the $\mathrm{UK}$, for instance, only accredited colonoscopy centers are allowed to participate in major programs. In Germany, infraqualified endoscopists are restricted from participating. Other examples abound.

Nevertheless, quality is not easy to achieve. Referring specifically to endoscopic screening approaches, there are a number of problems that are currently surfacing as more strict outcome evaluation becomes the norm. A worrisome problem now much talked about concerns missing significant precancerous lesions, particularly in the right colon [9]. The problem is compounded by the fact that some right colonic lesions are not only more difficult to detect but they carry an increased risk of malignant degeneration. Particularly relevant among these are sessile depressed lesions that are now recognized as being particularly prone to develop high-grade dysplasia or invasive carcinoma. Also, serrated polyps are likely to incorporate MSI (microsatellite instability) and CIMP (CpG island methylator phenotype) which confer a high probability of evolving into carcinoma [10].

Quality may also be affected by practical aspects unrelated to pathology. As I previously alluded to, the limited capacity of medical systems to deal with high colonoscopy loads may also interfere with efforts to improve quality. In the USA, for instance, the Veterans Administration programmatic screening [11] has at some point generated demand exceeding available capacity, which compromises the quality of the whole program by challenging its ability to comply with colonoscopy interval targets.

Colon screening modalities, other than colonoscopy, may be hampered by quality issues. The fecal immunochemical tests that are commercially available have widely different specificities and, therefore, results are influenced by the test employed. CT colography, despite early enthusiasm as a potential replacement for colonoscopy, has raised substantial cost-effectiveness concerns including the potential the problem of extracolonic findings and how to evaluate and manage them. CT colography, in the elderly particularly, also has to resolve how to deal with the frequent finding of polyps and which criteria to follow in either observing or removing them [12]. Although some studies suggest that small polyps may be safely ignored, there is no doubt that in some instances even diminutive lesions may harbor high-risk aberrant tissue [13].

Capsule endoscopy is another relatively novel screening method that is credited with higher patient acceptability that either colonoscopy or CT colography. Unfortunately, capsule endoscopy still has not achieved the necessary sensitivity to make it widely accepted as a valid screening method for colonic neoplasia. It is also true, however, that capsule endoscopy keeps technically improving and in the not so distant future could become an extremely competitive technique.

The future, at least in theory, belongs to serum tests. These may eventually achieve the required specificity and sensitivity to make them the preferred screening method to both physicians and patients. At the present time, their specificity is already reasonably good (for instance, the serum hypermethylation test of the Septin 9 gene already achieves a specificity of over $90 \%$ ) but the sensitivity of these serological tests remains discouragingly low. Projections, however, indicate that in the long range these sensitivity problems will be overcome and that serum tests with become the favorite screening modality by the general population and physicians alike.

\section{The Barrett's Quagmire}

Unlike cancer of the colon, esophageal adenocarcinoma is a relatively uncommon neoplasia. But its incidence is growing, a phenomenon that has been purportedly linked to an increased prevalence of gastroesophageal reflux in the Western general population, itself perhaps on account of unhealthy eating habits and overweight. Barrett's esophagus, a condition that affects up to $1-2 \%$ of individuals with symptomatic chronic gastroesophageal reflux, is a premalignant condition. Estimates as to the incidence of esophageal cancer (mostly adenocarcinoma) in patients with Barrett's esophagus hover around 0.5\% per year. High-grade dysplasia, premonitory of malignant transformation, has an annual incidence of 5-8\% among Barrett's patients. Considering these figures, is 
prevention of esophageal adenocarcinoma in the general population accomplishable, affordable, generalizable and reasonable to undertake?

At present, the answer to the above question is fraught with uncertainties and must be pondered with considerable caution. We certainly have various preventive instruments at our disposal: screening, surveillance plus preemptive ablation when feasible and chemoprevention. But each of these approaches carries associated challenges.

Let us consider first, screening. Epidemiological data brings to the forefront the magnitude and great cost of the task. Thus, the prevalence of weekly reflux symptoms in the general population is $10-15 \%$ and Barrett's esophagus may be found in about $8 \%$ of these symptomatic refluxers. Therefore, it would be necessary to screen 1 in 6 to 1 in 10 individuals to identify Barrett's and subsequently follow them up, to identify the small minority who would develop high-grade dysplasia and thus be at high risk for malignancy [14]. The challenge is magnified by the fact that Barrett's esophagus may be present in symptomless individuals, as shown by the incidental finding of Barrett's in up to $6 \%$ of endoscoped individuals who are investigated for reasons other than reflux symptoms. Moreover, a massive screening program would face the lingering controversy as to what constitutes premalignant Barrett's, since there may be hitherto unrecognized tissue characteristics in the predysplastic stage that determine whether or not some will progress towards neoplastic transformation. Relevance of length is another element in discussion, as well as the significance, in terms of neoplastic potential, of intestinal metaplasia versus absence of intestinal metaplasia or complete vs. incomplete intestinal metaplasia. To further complicate matters, patchy histology is relatively common when biopsy sampling is applied [15] and newer technologies including optical histology and other enhancement methods are still in the clinical evaluation phase, since they have not proven clear superiority to white light endoscopy, yet. Another relevant aspect is that studies to date have not conclusively established that generalized screening for detection of Barrett's esophagus would lower mortality.

If we discard generalized screening as too expensive and of uncertain value, surveillance of known Barrett's would seem the logical next step in the preventive strategy ladder, but there are problems associated with this approach as well. Surveillance would strictly apply to already diagnosed Barrett's with the aim of first detecting the appearance of high-grade dysplasia or early cancer, amenable to 'for cure' resection (surgical or endoscopic).
Unfortunately, although it has been reasonably well established that the degree of dysplasia positively correlates with cancer risk, there is considerable variability in the identification and grading of dysplasia in Barrett's esophagus [16]. The initial degree of dysplasia in itself is a relatively weak predictor of subsequent grade progression. That is, the rate of change from low- to high-grade dysplasia may be partially offset by the rate of disappearance of low-grade dysplasia - the regression rate being in itself variably influenced by other factors such as antisecretory therapy. Again, as I pointed out for screening, it has not been possible to demonstrate that surveillance reduces mortality from adenocarcinoma arising from Barrett's esophagus.

Notwithstanding the above provisos, surveillance of Barrett's esophagus is likely to approach its theoretical marks in the future on account of technological advances rapidly taking place, especially in two areas. First, novel endoscopic imaging techniques are increasingly capable to surf the Barrett's mucosal surface. As technology improves, these methods may become more accurate and efficient to pick up relevant tissue anomalies. Second, better biomarkers are looming in the future as an (as yet unfulfilled) promise. Furthermore, the effectiveness of surveillance is constantly strengthened by improvements in therapeutic techniques that facilitate ablation of the metaplastic issue at risk [17]. On this count, both endoscopic mucosal resection and radiofrequency ablation (HALO) are proving increasingly valuable and it has already been shown, reassuringly, that under proton pump inhibitor (PPI) protection, the esophageal mucosa postablation tends to regenerate in its original stratified structure $[18,19]$.

Chemoprevention is another appealing approach to avert malignant transformation of Barrett's tissue, but progress in this field has been relatively modest so far. PPIs are already commonly used in symptomatic Barrett's to improve patient's quality of life. PPI therapy may achieve in some patients partial regression of metaplastic mucosa, but the effect is insufficient to constitute a reliable ablation approach [20]. PPI therapy, however, may be associated with decreased progression to high-grade dysplasia and mucosal adenocarcinoma, according to some studies. This advantageous effect is supported by other trial data indicating that surveillance new entries of individuals on PPIs are less likely to harbor low-grade dysplasia than individuals without PPI treatment [21]. Antireflux procedures may share this protective effect with PPI maintenance treatment. Although antireflux surgery has not proven protective for adenocarcinoma of the esopha- 


\begin{tabular}{|ll|}
\hline Predictive & $\begin{array}{l}\text { To identify what conditions an individual } \\
\text { may develop in the future }\end{array}$ \\
\hline Preventive & $\begin{array}{l}\text { To facilitate a proactive approach to } \\
\text { health and medicine by shifting the } \\
\text { focus from illness to wellness }\end{array}$ \\
\hline Participatory & $\begin{array}{l}\text { To empower patients to make informed } \\
\text { choices and assume responsibility for } \\
\text { their own health }\end{array}$ \\
\hline
\end{tabular}

Fig. 4. The science of personalized medicine.

gus, some studies show a trend towards reduction of dysplasia and cancer risk. Finally, some studies suggest that low-dose ASA confers some protection, particularly if associated with PPI therapy, but these results need to be confirmed and expanded before ASA chemoprevention is recommended as standard practice.

\section{Prevention in the Era of Personalized Medicine}

Personalized medicine is already upon us and must be counted as an important player in the prevention of gastrointestinal disease. Personalized medicine is expected to enable tailored approaches to prevention by leveraging the science of genomics and proteomics. Personalized medicine may be considered predictive in that it aims to identify conditions an apparently healthy individual may develop in the future. It is also preventive in that it facilitates a proactive approach to health and medicine, because it shifts the focus from illness to wellness. It is also participatory as it empowers individuals to make informed choices and to assume responsibility for their own health (fig. 4).

Personalized medicine rests on two major elements of support: genetic testing and electronic health records. Genetic testing is already a booming market in the USA and other advanced countries. Interestingly, and perhaps worryingly, the fastest growth is in direct to consumer testing! This is not that surprising considering the enormous interest that genetic advances and genetic testing in particular have generated in the media including, of course, the web, the printed media and the waves. Genetic testing is also well suited to commercial development by private laboratories and increasingly affordable. Reflect, as an example, on the fact that it took 13 years and USD 2.5 billion to complete the human genome proj- ect in 2003. Compare these figures with the projected cost of about USD 100 to replicate it individually in $15 \mathrm{~min}$ in the very near future! However, such rapid growth and increased accessibility raises some important issues. On the one hand, the precise biological mechanisms that predict or trigger disease in a given individual remain largely unelucidated. It is also relevant to express our relative ignorance as to the interaction of multiple genes and proteins with the environment and how it affects the predictability of genetic data vis-à-vis the future development of disease. At the very least, translating genetic knowledge into efficient preventive strategies remains, in practical terms, a complex task. The role of physicians in the process is also critical, since without guidelines and competent counselors there is a significant risk that widespread genetic testing may simply add cost to an already financially strained health system, with no clear understanding of the results. In this regard, it has been pointed out that physicians, in the not so distant future, risk being desintermediated unless they quickly acquire the engineering/ computing skills that may be necessary in dealing with large quantities of genetic data.

The importance of the electronic health record arm of the equation is also easy to grasp. Electronic health records are already being implemented and to some extent enforced, because of their importance in fostering standardized health delivery and to improve the efficiency and economic accountability of medical settings. To comprehend the real significance of genetic data, it will be necessary to match large quantities of genomic and proteomic data with clinical information stored electronically. This will allow recognition of clinically useful patterns of pathologic genotype-phenotype coupling eventually allowing individualization of care. A fully developed electronic health system is the only way to efficiently do such matching. The road is long and difficult, but the race has already started.

\section{Disclosure Statement}

No conflict of interest. 


\section{References}

1 Johanson JF (ed): Risk Factors and Prevention. Philadelphia, Lippincott-Raven, 1997, pp 296.

12 Kirkegaard H, Johnsen NF, Christensen J, et al: Association of adherence to lifestyle recommendations and risk of colorectal cancer: a prospective Danish cohort study. BMJ 2010;341:e-5504.

-3 Rothwell PM, Wilson M, Elwin CF, et al: Long-term effect of aspirin on colorectal cancer incidence and mortality: 20-year follow-up of five randomised trials. Lancet 2010;376:1741-50.

-4 Baxter NN, Goldwasser MA, Paszat LF, et al: Association of colonoscopy and death from colorectal cancer. Ann Intern Med 2009;150: $1-8$.

5 Levin B, Lieberman DA, McFarland B, et al: Screening and surveillance for detection of colorectal cancer and adenomatous polyps, 2008: a joint guideline from the American Cancer Society, the Multi-Society Task Force on Colorectal Cancer, and the American College of Radiology. Gastroenterology 2008;134:1570-1595.

6 US Preventive Services Task Force: Screening for colorectal cancer: US Preventive Services Task Force recommendation statement. Ann Intern Med 2008;149:627-37.
7 Hoff G, Dominitz JA: Contrasting US and European approaches to colorectal cancer screening: which is best? Gut 2010;59:407414.

8 Malila N, Oivanen T, Malminiemi O, et al: Colorectal cancer screening in Finland: details of the national screening programme implemented in Autumn 2004. J Med Screen 2005;112:28-32.

$\checkmark 9$ Mitchal A, Schreiner, et al: Proximal and large hyperplastic and nondysplastic serrated polyps detected by colonoscopy are associated with neoplasia. Gastroenterology 2010;139:1497-1502.

10 Hiraoka S, Kato J, Fujiki S, et al: The presence of large serrated polyps increases. Gastroenterology 2010;139:1503-1510.

11 Kussman MJ: VHA Directive 2007-004. Colorectal Cancer Screening. Washington, Department of Veterans Affairs, Veterans Health Administration, 2007.

12 Odze RD: A balancing view: pathologist/clinician interaction is essential. Am J Gastroenterol 2008;103:1331-1333.

13 Brenner H, Hoffmeister M, Arndt V, et al: Protection from right- and left-sided colorec tal neoplasms after colonoscopy: population-based study. J Natl Cancer Inst 2010; 102:89-95.

14 Lagergren J, Ye W, Bergström R, et al: Utility of endoscopic screening for upper gastrointestinal adenocarcinoma. JAMA 2000;284 961-962.
15 Jones TF, Sharma P, Daaboul B, et al: Yield of intestinal metaplasia in patients with suspected short-segment Barrett's esophagus on repeat endoscopy. Dig Dis Sci 2002;47:21082111.

16 Odze RD, Maley CC: Neoplasia without dysplasia: lessons from Barrett's esophagus and other tubal gut neoplasms. Arch Pathol Lab Med 2010;134:896-906

17 Gress FG: Endoscopic Management of Barrett's Esophagus. Clinical Update. Oak Brook/IL, American Society for Gastrointestinal Endoscopy, 2009, p16.

18 Inadoni JM, Somsouk M, Madanick RD: A cost-utility analysis of ablative therapy for Barrett's esophagus. Gastroenterology 2009; 136:2101-2114.

19 Das A, Wells C, Kim HJ: An economic analysis of endoscopic ablative therapy for management of nondysplastic Barrett's esophagus. Endoscopy 2009;41:400-408.

20 Peters FTM, Ganesh S, Kuipers EJ: Endoscopic regression of Barrett's oesophagus during omeprazole treatment; a randomised double-blind study. Gut 1999;45:489-494.

21 El-Serag HB, Aguirre TV, Davis S: Proton pump inhibitors are associated with reduced incidence of dysplasia in Barrett's esophagus. Am J Gastroenterol 2004;99:1877-1883. 